\title{
Carcinomatous-like mastitis due to axillary lymphadenopathy in a case of nasopharyngeal carcinoma: A case report
}

\author{
CRISTINA MARINELA OPREAN ${ }^{1-3}$, NUSA ALINA SEGARCEANU ${ }^{2,3}$, ALEXANDRA STAN $^{4}$, \\ CRISTIAN SILVIU SUCIU ${ }^{5}$, DACIANA GRUJIC ${ }^{6,7}$, IOANA ALEXANDRA RIVIS ${ }^{8,9}$, \\ ALIS LILIANA CARMEN DEMA $^{10}$ and ANA CRISTINA BREDICEAN ${ }^{9,11}$
}

\begin{abstract}
${ }^{1}$ Discipline of Morpho-Pathology, 'Victor Babes' University of Medicine and Pharmacy of Timisoara, 300041 Timisoara; ${ }^{2}$ Department of Oncology, OncoHelp Hospital; ${ }^{3}$ Department of Oncology, Oncomed Outpatient Unit, 300239 Timisoara;

${ }^{4}$ Department of Oncology, Emergency City Hospital, 300254 Timisoara; ${ }^{5}$ Discipline of Histology, 'Victor Babes' University of Medicine and Pharmacy of Timisoara; 'Department of Plastic and Reconstructive Surgery, 'Victor Babes' University of Medicine and Pharmacy of Timisoara; ${ }^{7}$ Clinic of Burns, Plastic and Reconstructive Surgery, 'Pius Branzeu' Emergency County Hospital, 300041 Timisoara; ${ }^{8}$ Neurosciences Department, 'Carol Davila' University of Medicine and Pharmacy, 020021 Bucharest; ${ }^{9}$ NEUROPSY-COG Center for Cognitive Research in Neuropsychiatric Pathology, Neurosciences Department, 'Victor Babes' University of Medicine and Pharmacy of Timisoara;

${ }^{10}$ ANAPATMOL Research Center, 'Victor Babes' University of Medicine and Pharmacy of Timisoara, 300041 Timisoara; ${ }^{11}$ Psychiatry Compartment, 'Dr Victor Popescu’ Emergency Hospital, 300080 Timisoara, Romania
\end{abstract}

Received April 29, 2021; Accepted June 1, 2021

DOI: $10.3892 /$ etm.2021.10458

\begin{abstract}
Correspondence to: Professor Alis Liliana Carmen Dema, ANAPATMOL Research Center, 'Victor Babes' University of Medicine and Pharmacy of Timisoara, Piata Eftimie Murgu 2, 300041 Timisoara, Romania

E-mail: dema_alis@yahoo.com

Dr Ioana Alexandra Rivis, Neurosciences Department, 'Carol Davila' University of Medicine and Pharmacy, 37 Dionisie Lupu Street, 020021 Bucharest, Romania

E-mail: ioana.rivis@gmail.com
\end{abstract}

Abbreviations: TPF, docetaxel, cisplatin, and 5-fluorouracil; NPC, nasopharyngeal carcinoma; HNSCC, squamous cell carcinoma of the head and neck; NP, nasopharynx; NSCC, non-keratinizing squamous cell carcinoma; SCC, squamous cell carcinoma; DFI, disease-free interval; CR, complete response; IHC, immunohistochemistry; PR, partial response; CT, computed tomography; Gy, gray; GCSF, granulocyte colony-stimulating factor; ENT, ear-nose-throat; CK, cytokeratin; HMWCK, high-molecular-weight cytokeratin; EMA, epithelial membrane antigen; CgA, chromogranin A; Syn, synaptophysin; GATA-3, trans-acting T cell-specific transcription factor; CD10, neutral peptidase 24.11 (NEP); SMA, smooth muscle actin; TTF1, thyroid transcription factor 1; DAB, diaminobenzidine; EBV, Epstein-Barr virus; EA, early antigen; Ig, immunoglobulin; EBNA, Epstein-Barr nuclear antigen; VCA, virus capsid antigen; EBER, Epstein-Barr encoding region; RTU, ready-to-use; HIER, heat induced epitope retrieval; HRP, horseradish peroxidase; PCR, polymerase chain reaction; DNA, deoxyribonucleic acid; NGS, next generation sequencing; $\mathrm{H} \& \mathrm{E}$, hematoxylin and eosin

Key words: head and neck cancer, nasopharyngeal carcinoma, axillary lymph node metastasis, carcinomatous mastitis, case report
Abstract. Nasopharyngeal carcinoma (NPC) is a rare form of malignancy, accounting for $2 \%$ of all cancers of the head and neck in Europe. Axillary lymph node metastases are very rare in these cases. This is a case report of a 40 -year-old premenopausal woman diagnosed in May 2015 with T1N2M0 stage III NPC, treated with induction chemotherapy, followed by chemo-radiotherapy. Post-therapeutic computed tomography $(\mathrm{CT})$ scan showed partial response (PR) on the primary tumor and complete response (CR) on the latero-cervical lymph nodes. In 2017, our patient developed left carcinomatous-like mastitis with axillary lymphadenopathy. This raised suspicions of a carcinomatous mastitis. The pathology report with immunohistochemistry (IHC) of the third biopsy highlighted axillary metastasis of a non-keratinizing squamous cell carcinoma (NSCC). There are very few references in the literature regarding axillary metastases from squamous cell carcinoma of the head and neck (HNSCC). As far as we know, this is the first case report of mastitis due to NPC. To conclude, treatment consisted of two surgical excisions of axillary lymphadenopathy associated with local radiotherapy and chemotherapy (neo-adjuvant, adjuvant). The second surgery, performed after radiotherapy, required plastic surgery. A psychiatric evaluation was necessary, revealing a reactive anxiety disorder. This case required multidisciplinary management, where oncology, plastic surgery, pathology and psychiatric specialists collaborated in deciding the therapeutic approach.

\section{Introduction}

Nasopharyngeal carcinoma (NPC) is the most common type of cancer in southern Asia (1,2). In Europe, its incidence is rare, with an annual rate varying between 2.1 and 0.4 per 100,000 individuals (3-5). NPC represents $2 \%$ of all cancers developed 
in the head and neck (6). The incidence is higher in men than in women with an M:F ratio of $2.75(3,4)$. Epstein-Barr virus infection is also a factor in most neck and head cancer cases, along with smoking and alcohol consumption (7-9). Relative survival for NPC in Europe is $49 \%$ at 5 years, with no differences in survival between sexes (3). HNSCC has been proven to distantly spread below the clavicles (10). The chance of dissemination is related to the cervical lymph node involvement. For N0-N2 tumors, the dissemination occurs in less than $10 \%$ of cases compared with $30 \%$ in N2-N3 cases (10).

The lymphatic drainage from the head and neck occurs through a superficial and deep system. The superficial group of cervical lymph nodes includes occipital, postauricular, parotid, facial, submandibular, sub-mental and superficial cervical nodes, which are situated alongside the external jugular vein. The profound group of cervical lymph nodes lies along the internal jugular vein. All of the lymphatic vessels of the head and neck empty into the deep group, either directly or via the superficial group of nodes (11). The lymphatic system, within the axilla region, normally flows from the distal portion of the upper limb and the chest wall along the axillary vein toward the subclavian venous system (12).

Like most other locations of HNSCC, the lymphatic drainage of the nasopharynx (NP) is predominantly directed to the cervical lymph nodes $(13,14)$. Lymph node metastases are present in $80 \%$ of NPC cases, and in $50 \%$ of the cases, these are bilateral. The worst aspect of cancer is its ability to spread or metastasize (15). Patients with lymph node metastases have a more favorable prognosis than those with visceral metastases (16). It is known that NPC tumors are associated with a high incidence of distant metastases $(17,18)$. Pulmonary metastases represent the most common localization, accounting for $66 \%$ of total distant metastases. Other metastatic sites include bone (22\%) and liver (10\%) (18-20). Axillary lymph node metastasis is an uncommon event in HNSCC. There are very few references in the literature regarding axillary metastases from HNSCC (21). Only five reports have been published in the literature, including 10 patients (22). None of these cases included a co-occurrence of mastitis. Axillary metastases are mentioned in only 2 of 8 published autopsy studies (10). Upon autopsy reports, between 2 to $9 \%$ of patients with HNSCC tumors were found to have axillary node metastases, but it is assumed that the incidence is higher, as impalpable lymph nodes are not routinely examined during autopsy, except in breast cancer cases and in some malignant melanomas $(23,24)$.

\section{Case report}

We present the case of a 40-year-old woman, a stay-at-home mother of two children (aged 5 and 8), who lives in the rural area, with no family history of neoplasm or any other comorbidities. The onset of the disease was in April 2015, when our patient started noticing a nasopharyngeal discomfort, nasal obstruction, rhinorrhea and epistaxis. The ear-nose-throat (ENT) exam revealed a lesion of $2.3 / 2 \mathrm{~cm}$ in the NP, for which a biopsy was completed. Clinically, the patient presented bilateral cervical lymphadenopathy with diameters of up to $3.5 \mathrm{~cm}$. In May 2015, the diagnosis was confirmed: Stage III, T1N2M0 poorly differentiated NSCC of the NP.
By November 2015, the patient had undergone 3 cycles of docetaxel, cisplatin, and 5-fluorouracil (TPF) induction chemotherapy associated with prophylactic supportive granulocyte colony-stimulating factor (GCSF) treatment, with PR after induction chemotherapy, followed by concomitant weekly cisplatin $\left(40 \mathrm{mg} / \mathrm{m}^{2}\right)$ radio-chemotherapy $66 \mathrm{Gray}(\mathrm{Gy})$ on the NP, 58 Gy on the bilateral cervical lymph nodes and 50 Gy on the bilateral supraclavicular areas. Both treatment adherence and tolerance were satisfactory. The post therapeutic brain CT scan, performed in March 2016, showed PR at the primary NPC, and CR at the latero-cervical lymph nodes.

On February 2017, the patient reported pain, edema, erythema and swelling of the left breast. The physical examination noted left axillary lymphadenopathy and left-sided mastitis. In order to explore the probability of a coexistent breast cancer (carcinomatous mastitis), an excisional biopsy was performed on the left axillary lymph nodes, which excluded a neoplastic process. The patient received local and oral treatment with anti-inflammatory drugs (300 mg of ketoprofen per day) and antibiotics (amoxycillin/clavulanic acid $1,000 \mathrm{mg} / 62.5 \mathrm{mg}$, twice a day) for 10 days, after which the mastitis went into slight remission for the next 4 months.

In June 2017, the inflammation of the left breast reoccurred along with the worsening of the previous symptoms. Chest CT scan confirmed left axillary (of $4.5 \mathrm{~cm}$ ) and supraclavicular lymph node enlargement (of $3.5 \mathrm{~cm}$ ), and left mastitis. Left axillary lymph node excisional biopsy was performed after 10 days of systemic antibiotic and anti-inflammatory treatment. The pathology report revealed another inflammatory reaction of the lymph node, without tumor infiltration.

In September 2017, due to the persistence of the clinical signs of mastitis, despite oral antibiotic and anti-inflammatory treatment, the patient attended our clinic looking for a second medical opinion. Clinical examination revealed severe mastitis, lymphedema of the left arm and lymphadenopathy in the left axilla and supraclavicular region (Fig. 1). A new subcutaneous lesion on the left axilla and thorax was discovered. The total body CT scan showed a progressive aspect of mastitis and an increase in the axillary and supraclavicular lymph nodes (Figs. 2 and 3).

The primary NPC tumor and latero-cervical lymph nodes were in CR. One subcutaneous nodule from the axillary left region was excised and sent for pathological examination. The pathology report described large islands of tumor cells with prominent nucleoli and admixed inflammatory cells (Fig. 4). IHC staining was done on paraffin-embedded tissue, with the following antibodies: Trans-acting T cell-specific transcription factor (GATA-3), mammaglobin, epithelial membrane antigen (EMA), cytokeratin (CK) AE1/AE3, CK5, high-molecular-weight cytokeratin (HMWCK) (34BE12), CK7, p63, neutral peptidase 24.11 (NEP) (CD10), S100, smooth muscle actin (SMA), p16, chromogranin A (CgA), synaptophysin (Syn). Serial sections, 3- $\mu \mathrm{m}$ thick, were constructed from one selected paraffin-block with the representative tumor area, and then mounted on silanized slides, so as to prevent detachment during antigen unmasking procedures. Tissue sections were deparaffined in xylene and rehydrated. For unmasking the antigens, we used heat induced epitope retrieval (HIER) by boiling the slides for $20 \mathrm{~min}$ at $98^{\circ} \mathrm{C}$ in target retrieval solution $\mathrm{pH} 6.0$ or 9.0. Endogenous peroxidase activity was blocked 


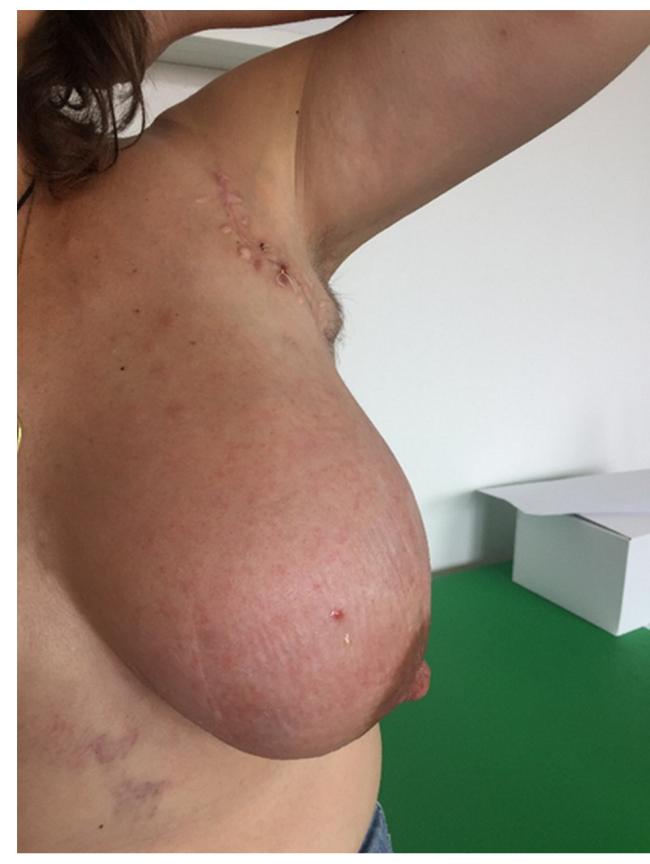

Figure 1. Left breast mastitis.

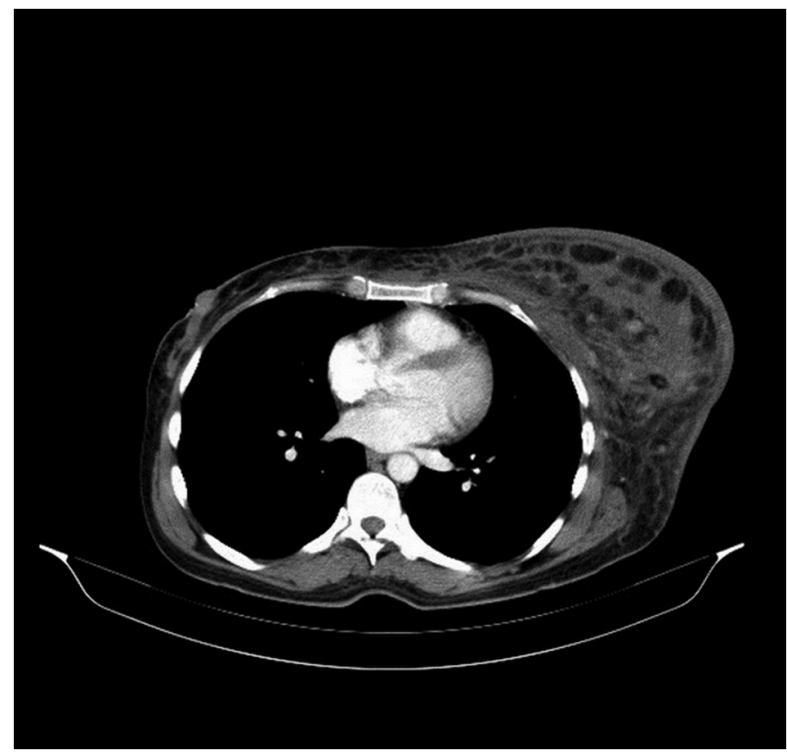

Figure 2. Left breast mastitis on CT (September 2017). CT, computed tomography.

by treatment with $0.5 \% \mathrm{H}_{2} \mathrm{O}_{2}$ for $15 \mathrm{~min}$. Subsequently, we applied ready-to-use (RTU) or concentrated antibodies. For the concentrated antibodies, the slides were incubated overnight in a refrigerator at $4^{\circ} \mathrm{C}$ with appropriate dilutions of the primary antibodies. The reaction was visualized by Novolink kit-HRP (horseradish peroxidase) with diaminobenzidine (DAB) as chromogen. Finally, the sections were counterstained with hematoxylin, dehydrated, clarified and mounted with Entellan. The tumor cells presented the following IHC profile: Extensive expression for p63(+++) (Fig. 5), less extensive reactivity for HMWCK $(++)$ (Fig. 6), limited expression for CK8/18(+), negative reaction for CK7, GATA-3, thyroid transcription factor 1 (TTF1) and S100. Serum antibodies against Epstein

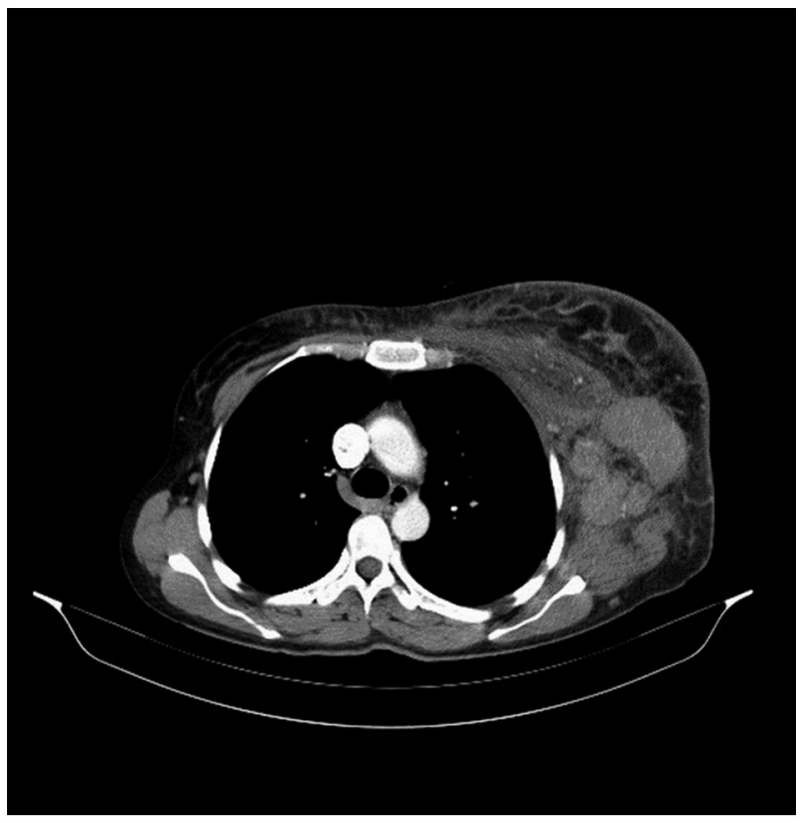

Figure 3. Left breast mastitis and axillary lymph nodes on CT (September 2017). CT, computed tomography.

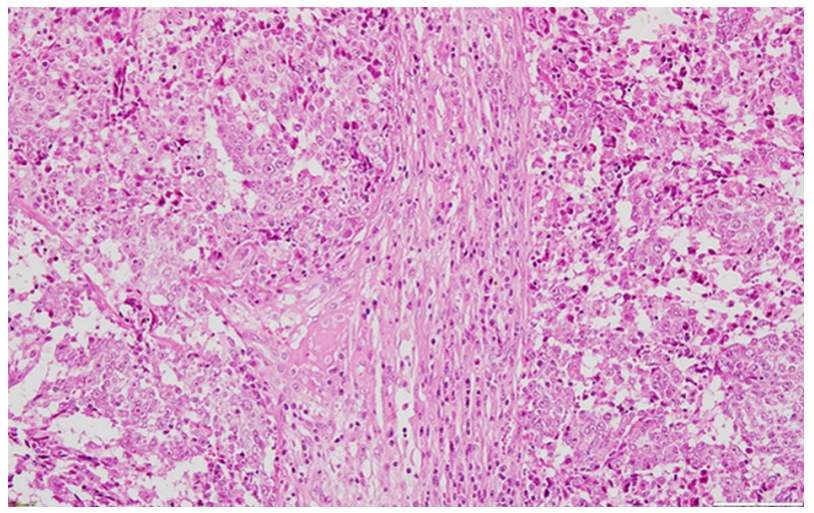

Figure 4. Large islands of tumor cells with prominent nucleoli and admixed inflammatory cells. H\&E, x 200. H\&E, hematoxylin and eosin.

Barr virus (EBV) were investigated. Early antigen (EA) immunoglobulin (Ig) $\mathrm{G}$ was positive ( $>150 \mathrm{U} / \mathrm{ml})$; antibodies against Epstein-Barr nuclear antigen (EBNA) IgG were highly positive $(>600 \mathrm{U} / \mathrm{ml})$, virus capsid antigen (VCA) IgM antibodies were negative $(<10.0 \mathrm{U} / \mathrm{ml})$. Blood deoxyribonucleic acid (DNA) EBV quantitative polymerase chain reaction (PCR) was undetectable. Unfortunately, the in situ hybridization for Epstein-Barr encoding region (EBER) was not able to be performed. Based on morphological and IHC data, the tumor was diagnosed as metastasis of an NSCC, most probably from an NPC, based on the positive serology for the Epstein-Barr virus.

In association to all of the above, our patient presented anxiety and sleeping difficulties, especially when it came to the initiation of sleep. Therefore, a psychiatric evaluation was necessary, which revealed a reactive anxiety disorder. She was prescribed medazepam $10 \mathrm{mg}$ per day, for four weeks, and six psychiatric counselling sessions. Gradually, the 


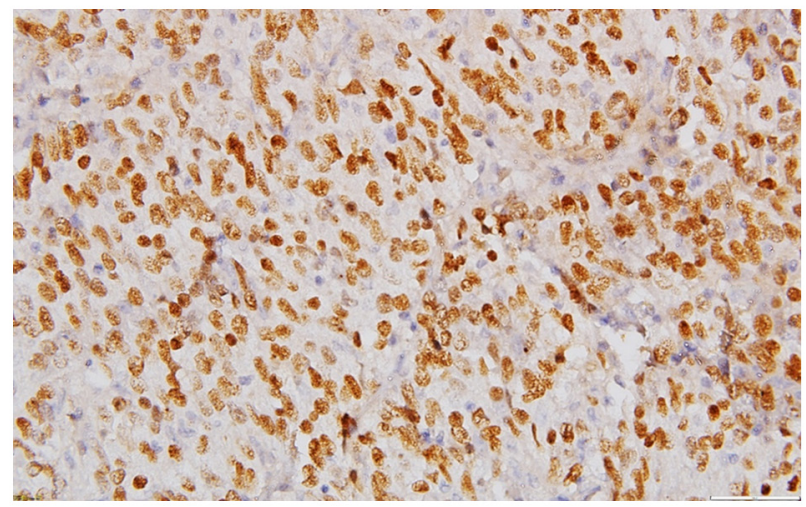

Figure 5. Extensive expression for p63 in the tumor cells. Anti-p63, DAB, counterstain with hematoxylin x 400. DAB, diaminobenzidine.

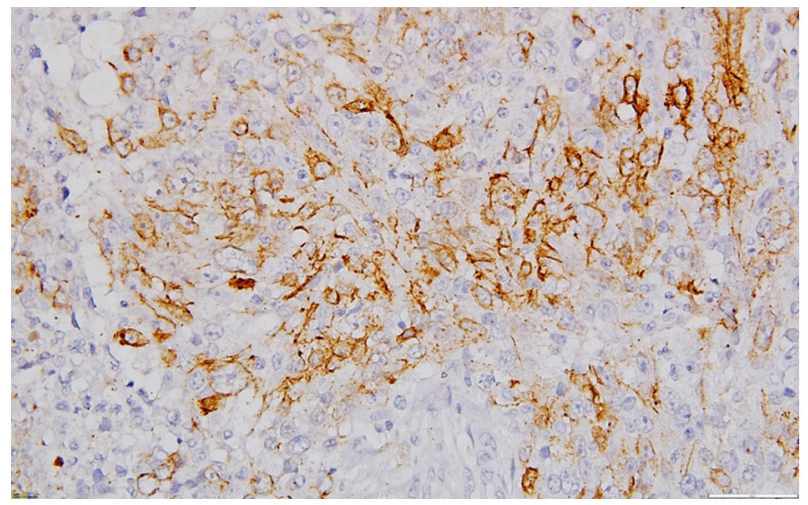

Figure 6. Less extensive reactivity for HMWCK (clone 34ßE12) in the tumor cells. Anti-HMWCK, DAB, counterstain with hematoxylin x400. HMWCK, high-molecular-weight cytokeratin; DAB, diaminobenzidine.

symptoms started to alleviate, and after a total of six weeks, they completely disappeared.

The patient started chemotherapy with cisplatin $\left(75 \mathrm{mg} / \mathrm{m}^{2}\right)$ and docetaxel $\left(75 \mathrm{mg} / \mathrm{m}^{2}\right)$ every 3 weeks, with GCSF support. After 4 cycles, in January 2018, the edema of the left breast and arm was in remission (Fig. 7). Clinically, the left axillary lymph nodes decreased and the supraclavicular ones disappeared. The patient had a mild cough, no fever, but an unpleasant fetid breath. Chest CT scan confirmed two pulmonary abscesses, PR of the left axillary lymph nodes and CR of the left supraclavicular nodes. Chemotherapy was postponed for two months, due to this severe lung infection. After remission, the same chemotherapy schedule was restarted and continued until cycle 11 .

In September 2018, full-body CT-scan showed persistence of CR at primary NPC and loco-regional lymph nodes, a mild diffuse edema of the left breast and a decrease in the left axillary lymph nodes (at $1.8 \mathrm{~cm}$ ) (Figs. 8 and 9). Afterwards, our patient underwent a surgical excision of the left axillary lymph nodes. The pathological report revealed the presence of 4 lymph nodes between 0.5 and $2 \mathrm{~cm}$, all of them metastatic NSCC, most probably originating in the NP. Our patient received adjuvant radiotherapy of $50 \mathrm{~Gy} / 25$ fractions on the axilla, until January 2019. The treatment was well-tolerated. After 9 months of disease-free interval (DFI), left breast mastitis and a left

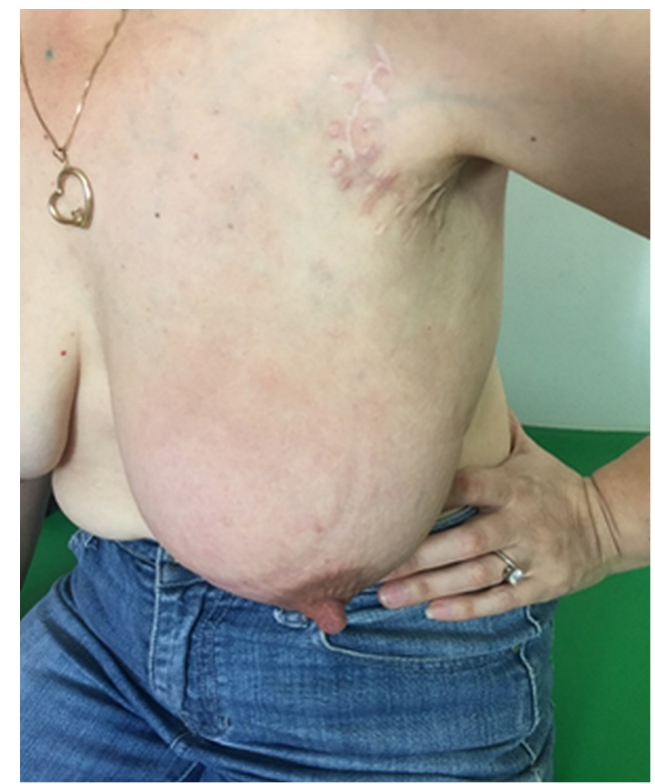

Figure 7. Remissive mastitis of the left breast after 4 cycles of chemotherapy (January 2018).

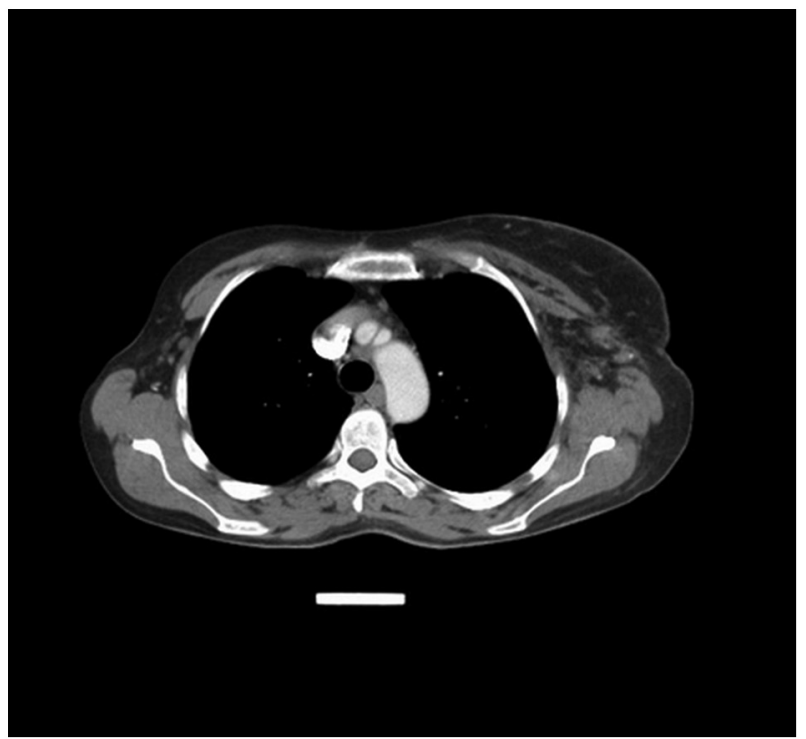

Figure 8. Mild diffuse edema of the left breast (September 2018).

axillary lymph node metastasis recurred. The clinical exam showed left breast mild mastitis and a mobile $3.3 \mathrm{~cm}$ axillary lymphadenopathy, which was programmed for excision.

The retractile scar-tissue was removed surgically, with a thorough dissection of the fibrous axillary tissue that resulted from scarring and radiotherapy, while isolating the lymphadenopathy block of the vascular pedicle and making a Z-plasty, in order to eliminate the adhesions. The pathology examination, including an extended IHC panel which found positive reaction for p63, CK5 (Fig. 10), CK AE1/AE3, focal reactivity for p16 (Fig. 11), cytoplasmic and nuclear and lack of reactivity for SMA, GATA-3, Syn, chromogranin A (CgA), CK7, EMA, S100, CD10, confirmed metastasis of a poorly differentiated NSCC. 


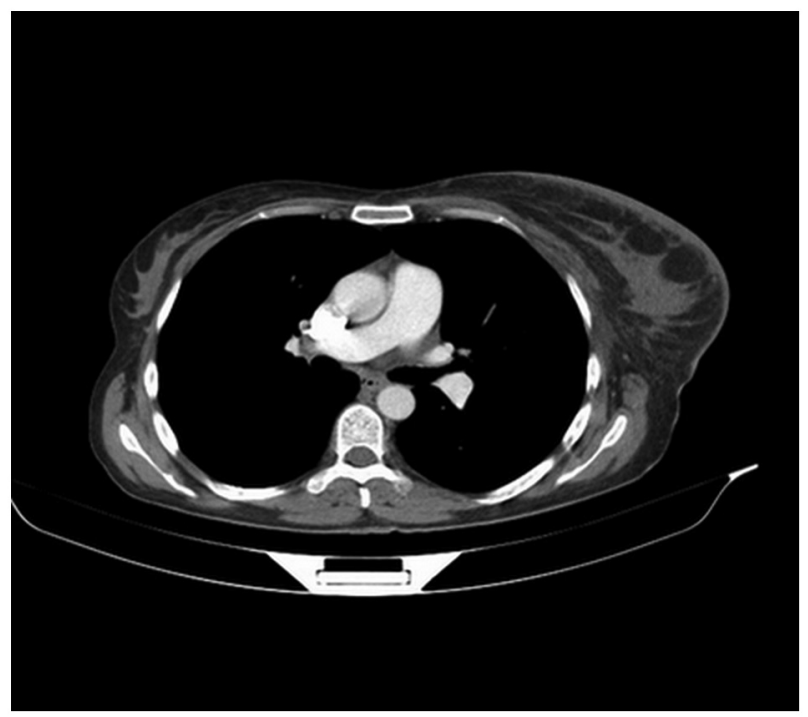

Figure 9. Left mastitis in remission and decrease of the left axillary lymph nodes (September 2018).

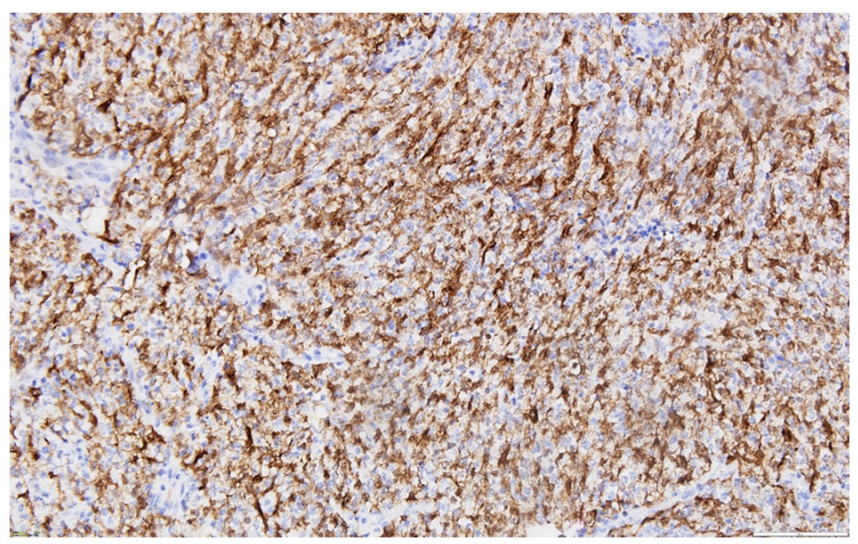

Figure 10. Diffuse expression of CK5 in the tumor cells. Anti-CK5, DAB, counterstain with hematoxylin, $x$ 200. CK, cytokeratin; DAB, diaminobenzidine.

The paraffin block from this last biopsy sample was used to test genomic alteration. The result showed NOTCH1 mutation-V1578 deletion. The patient was invited to be included in a phase I/II clinical trial abroad, but she refused. The pre-therapeutic total body CT scan evaluation did not highlight any other metastasis. Primary NPC tumor and loco-regional lymph nodes remained in $\mathrm{CR}$. The patient restarted chemotherapy with cisplatin and docetaxel in January 2020, with the same dosages and following the same schedule, as an 'adjuvant' treatment. On April 2020, the patient was undergoing chemotherapy treatment, and the clinical symptoms were in complete remission, but the upper left limb edema persisted (Fig. 12).

\section{Discussion}

Axillary lymph node involvement is rare in nasopharyngeal carcinoma (NPC) (21). Yet, they are a frequent site of regional metastatic disease from breast carcinoma (25). The recognition of axillary metastasis in patients with HNCC is crucial,

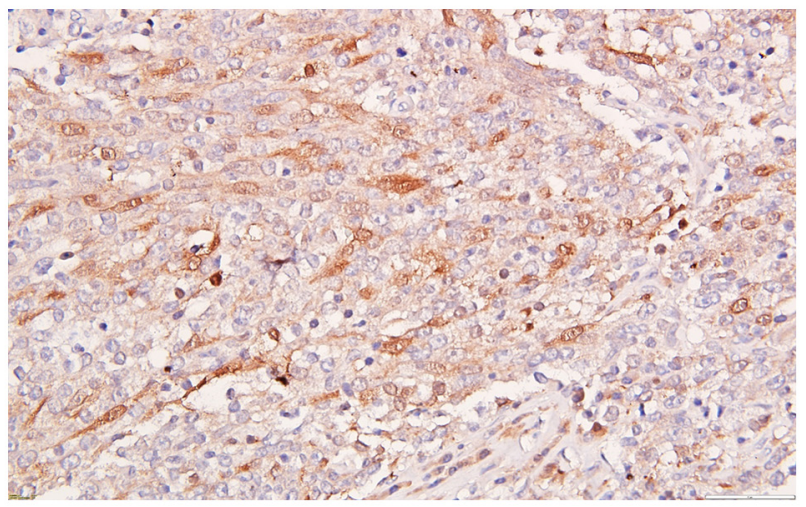

Figure 11. Focal reactivity for p16. Anti-p16, DAB, counterstain with hematoxylin, x400. DAB, diaminobenzidine.

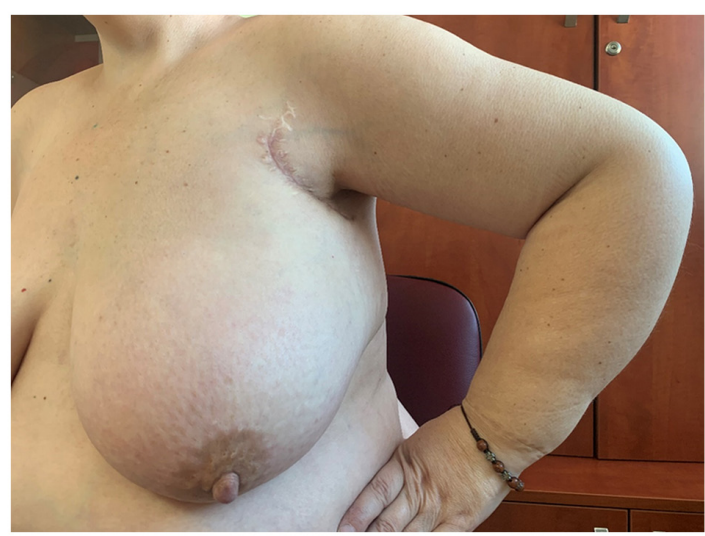

Figure 12. Local remission of left breast edema and persistent lymphedema of the left arm (April 2020).

especially in patients with massive metastases or delayed metastases at a low level of the head and neck (25). This article presents the case of a young patient, initially diagnosed and treated for NPC, who presented, in the evolution of the disease, axillary lymph node metastasis and carcinomatous-like mastitis. At the time of the axillary recurrence, the primary NPC had CR. Due to the clinical impression of the inflammatory breast carcinoma, we began by trying to diagnose a breast cancer, which was disproved following the results of the IHC and pathological examinations. Previous literature reports suggest that the involvement of axillary sites may be related to previous neck surgery, following a change in lymphatic drainage (11). Our patient had not undergone prior head and neck surgery.

The pathological aspects corresponded to a metastasis of a poorly differentiated/undifferentiated tumor, which is difficult to diagnose based on routine stained slides. The positive reaction for EMA and CK established the epithelial nature of the proliferation. Given the clinical aspect of inflammatory carcinoma combined with axillary lymphadenopathy, the IHC investigation, performed over two stages (2017 and 2019), was focused on removing the possibility of a metastasis from a second primary metachronous breast carcinoma.

The absence of CK7, mammaglobin and GATA3 reactivity, excluded the majority of most common breast carcinoma subtypes. The diffuse positivity for p63, CK5 and a 
heterogeneous one for HMWCK suggested a metastasis from a squamous or myoepithelial carcinoma, both of which are considered to be extremely rare subtypes of breast carcinoma. The absence of S100 and SMA reactivity excluded a breast lesion with myoepithelial differentiation. Knowing that p63 and HMWCK are positive in metaplastic breast carcinomas with spindle cells and/or squamous differentiation (26), the possibility that the axillary lesion represented a metastasis from these entities could not be completely ruled out. But considering what Wargotz and Norris (1990) affirmed, namely that before diagnosing a primary squamous cell carcinoma of the breast it is necessary to exclude a metastatic squamous cell carcinoma to the breast, the scenario of a metastasis from the previous diagnosed NPC was favored (27).

The main arguments for NPC metastasis against a metastatic metaplastic breast carcinoma with spindle cell and/or squamous differentiation included: An initial diagnosis of NPC; the important bilateral latero-cervical lymphadenopathy, staged cN2; the absence of a primary breast malignancy documented by imaging and excisional biopsies; the IHC profile of the axillary metastasis which overlaps with the nasopharyngeal lesion (conducted in another pathology department); the identification, by next generation sequencing (NGS), of NOTCH1 V1578 del and missense mutation of p53, when the NOTCH1 mutation is the second most common mutated gene (after TP53) in HNSCC (28); the positive serology for EBV, being well-known that nonkeratinizing NPC is associated with EBV in almost all cases (29).

Unfortunately, the in situ hybridization for EBER, a helpful investigation for establishing the NP as the primary site for a meta-virus capsid antigen static undifferentiated or poorly differentiated squamous cell carcinoma, could not be performed.

Emerging from the main genomic alteration identified, the NOTCH pathway, targeted therapy appeared to be a logical therapeutic option for this case. A theory regarding the favorable evolution of our patient may be due to, partly, the NOTCH1 mutation, since, more often than not, this mutation acts as a tumor-suppressor gene (30). The timely recovery surgery of the axillary metastasis after exclusion of other distant metastasis may improve the survival of these patients (25). HNSCC is one of the most aggressive malignancies (31). However, our patient had a survival of almost 5 years from the diagnosis of the primary tumor, and of about 3 years from the diagnosis of recurrence. For this patient, the surgical removal of the axillary lymph node was completed twice, for two local recurrences. We aimed to improve local control by adding radiotherapy of the axilla, after the first complete surgical excision. Chemotherapy was administered twice, in a 'neo-adjuvant' setting, first due to the initially inoperable recurrence, and second, in an 'adjuvant' setting, after the last surgery for the recurrence. In the absence of clear evidence on the efficacy of adjuvant therapy in this clinical situation, adjuvant chemotherapy was used for the second recurrence, which, although surgically removed, did not dismiss the possibility of systemic micro-metastasis, that cannot be identified by standard diagnostic methods. The correct duration of this present 'adjuvant' chemotherapy remains an open question. The role of local radiotherapy and systemic chemotherapy remains to be established in the future.
This particular case required a multidisciplinary management, where the oncology, plastic surgery, pathology and psychiatric medical specialists acted together in order to establish the correct diagnosis and adopt the appropriate therapeutic pathway. Currently, neoplastic patients benefit from personalized therapy, with the ultimate target of offering the patient a good quality of life.

\section{Acknowledgements}

The authors would like to thank the patient for allowing them to publish her case and to all the healthcare professionals who helped care for this patient.

\section{Funding}

No funding was received.

\section{Availability of data and materials}

Further information regarding this case report is available from the corresponding author on reasonable request.

\section{Authors' contributions}

CMO, NAS and AS were in charge of patient management, establishing the chemotherapy treatment and follow-up of the treatment response, and clinical evolution of the patient. ALCD and CSS were in charge of the laboratory diagnosis: Pathology, IHC and genomic testing. DG performed the two diagnostic interventions (biopsy) and the surgical axillary excision. ACB and IAR performed the psychiatric evaluation, established a treatment plan and offered psychological support. All authors read and approved the final manuscript for publication.

\section{Ethics approval and consent to participate}

For this case-report we obtained the patient's informed consent and her permission to use the images that were taken during her periodical check-ups. An ethical approval from the hospital was not required.

\section{Patient consent for publication}

Written informed consent was obtained from the patient regarding the publication of this case report and any accompanying images. A copy of the written consent is available for review.

\section{Competing interests}

The authors declare no competing interests.

\section{References}

1. Kuo DY, Chang MH, Wang SY, Hsieh PY and Shueng PW: Unusual axillary metastasis of recurrent nasopharyngeal cancer: A case report. Medicine (Baltimore) 96: e6854, 2017.

2. Mahdavifar N, Ghoncheh M, Mohammadian-Hafshejani A, Khosravi B and Salehiniya $\mathrm{H}$ : Epidemiology and inequality in the incidence and mortality of nasopharynx cancer in Asia. Osong Public Health Res Perspect 7: 360-372, 2016. 
3. Bossi P, Chan AT, Licitra L, Trama A, Orlandi E, Hui EP, Halámková J, Mattheis S, Baujat B, Hardillo J, et al: Nasopharyngeal carcinoma: ESMO-EURACAN clinical practice guidelines for diagnosis, treatment and follow-up $\dagger$. Ann Oncol 32: 452-465, 2021.

4. Parkin DM, Bray F, Ferlay J and Pisani P: Global cancer statistics, 2002. CA Cancer J Clin 55: 74-108, 2005.

5. Chang ET and Adami HO: The enigmatic epidemiology of nasopharyngeal carcinoma. Cancer Epidemiol Biomarkers Prev 15 1765-1777, 2006.

6. Zhou X, Cui J, Macias V, Kajdacsy-Balla AA, Ye H, Wang J and Rao PN: The progress on genetic analysis of nasopharyngeal carcinoma. Comp Funct Genomics 2007: 57513, 2007.

7. Fernandes Q, Merhi M, Raza A, Inchakalody VP, Abdelouahab N, Zar Gul AR, Uddin S and Dermime S: Role of Epstein-Barr virus in the pathogenesis of head and neck cancers and its potential as an immunotherapeutic target. Front Oncol 8: 257, 2018.

8. Pezzuto F, Buonaguro L, Caponigro F, Ionna F, Starita N, Annunziata C, Buonaguro FM and Tornesello ML: Update on head and neck cancer: Current knowledge on epidemiology, risk factors, molecular features and novel therapies. Oncology 89: 125-136, 2015.

9. Sturgis EM, Wei Q and Spitz MR: Descriptive epidemiology and risk factors for head and neck cancer. Semin Oncol 31: 726-733, 2004.

10. Nelson WR and Sisk M: Axillary metastases from carcinoma of the larynx: A 25-year survival. Head Neck 16: 83-87, 1994.

11. McKenzie BJ and Loock JW: Axillary nodal metastasis at primary presentation of an oropharyngeal primary carcinoma: A case report and review of the literature. J Med Case Rep 3 . 7230, 2009 .

12. Rayatt SS, Dancey AL, Fagan J and Srivastava S: Axillary metastases from recurrent oral carcinoma. Br J Oral Maxillofac Surg 42: 264-266, 2004.

13. Ho FC, Tham IW, Earnest A, Lee KM and Lu JJ: Patterns of regional lymph node metastasis of nasopharyngeal carcinoma: A meta-analysis of clinical evidence. BMC Cancer 12: 98, 2012.

14. Brennan B: Nasopharyngeal carcinoma. Orphanet J Rare Dis 1 23, 2006.

15. Dangore-Khasbage $S$ : Local metastasis in head and neck cancer-an overview. Contemporary Issues in Head and Neck Cancer Management. IntechOpen, pp152-167, 2015 https://doi.org/10.5772/60072. Accessed 8th July, 2015.

16. Alavi S, Namazie A, Sercarz JA, Wang MB and Blackwell KE: Distant lymphatic metastasis from head and neck cancer. Ann Otol Rhinol Laryngol 108: 860-863, 1999.

17. Chen MY, Jiang R, Guo L, Zou X, Liu Q, Sun R, Qiu F, Xia ZJ, Huang HQ, Zhang L, et al: Locoregional radiotherapy in patients with distant metastases of nasopharyngeal carcinoma at diagnosis. Chin J Cancer 32: 604-613, 2013
18. Tomao F, Miele E, Spinelli GP, Caprio G, Ranieri E, Mingazzini P, Zullo A and Tomao S: Axillary and subcutaneous breast metastases from rhinopharyngeal carcinoma: A case report and literature review. Anticancer Res 28: 419-424, 2008.

19. Chan AT, Teo PM and Johnson PJ: Nasopharyngeal carcinoma. Ann Oncol 13: 1007-1015, 2002.

20. Ahmad A and Stefani S: Distant metastases of nasopharyngeal carcinoma: A study of 256 male patients. J Surg Oncol 33: 194-197, 1986.

21. Koch WM: Axillary nodal metastases in head and neck cancer. Head Neck 21: 269-272, 1999.

22. Wormald R, Sheahan P and Timon C: A case of head and neck cancer metastasizing to the axillary lymph nodes. Ear Nose Throat J 89: E24-E26, 2010.

23. Islam S, Cole CV, Hoffman GR and Brennan PA: Bilateral axillary metastasis from a primary ethmoidal squamous cell carcinoma. J Laryngol Otol 120: 353-355, 2006.

24. Kowalski LP: Noncervical lymph node metastasis from head and neck cancer. ORL J Otorhinolaryngol Relat Spec 63: 252-255, 2001.

25. Oo AL, Yamaguchi S, Iwaki H and Amagasa T: Axillary nodal metastasis from oral and maxillofacial cancers: A report of 3 cases. J Oral Maxillofac Surg 62: 1019-1024, 2004.

26. Koker MM and Kleer CG: p63 expression in breast cancer: A highly sensitive and specific marker of metaplastic carcinoma. Am J Surg Pathol 28: 1506-1512, 2004.

27. Wargotz ES and Norris HJ: Metaplastic carcinomas of the breast. IV. Squamous cell carcinoma of ductal origin. Cancer 65: 272-276, 1990.

28. Fukusumi T and Califano JA: The notch pathway in head and neck squamous cell carcinoma. J Dent Res 97: 645-653, 2018.

29. El-Naggar AK, Chan JKC, Grandis JR, Takata T and Slootweg PJ (eds): WHO Classification of Head and Neck Tumours. Vol. 9. 4th edition. International Agency for Research on Cancer. IARC, Lyon, 2017.

30. Agrawal N, Frederick MJ, Pickering CR, Bettegowda C, Chang K, Li RJ, Fakhry C, Xie TX, Zhang J, Wang J, et al: Exome sequencing of head and neck squamous cell carcinoma reveals inactivating mutations in NOTCH1. Science 333: 1154-1157, 2011.

31. Solomon I, Voiculescu V, Caruntu C, Lupu M, Popa A, Ilie M, Albulescu R, Caruntu A, Tanase C, Constantin C, et al: Neuroendocrine factors and head and neck squamous cell carcinoma: An affair to remember. Dis Markers 2018: 9787831, 2018. International (CC BY-NC-ND 4.0) License. 